Molecules 2003, 8, 40-44

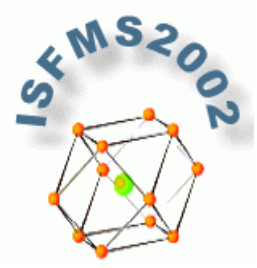

\title{
Sonochemical Degradation Kinetics of Methyl Violet in Aqueous Solutions
}

\author{
Xi Kui Wang*, Guan Hong Chen and Wei Lin Guo \\ College of Chemistry and Environmental Science, University of Jinan, Jinan 250022, Shandong \\ Province, P. R. China, Tel. (+86)-531-2765948, Fax (+86) 531-7150906 \\ * Author to whom correspondence should be addressed; e-mail: xk wang@ $x 263$.net
}

\begin{abstract}
The sonochemical degradation in aqueous solution of methyl violet, chosen as a model of a basic dye, was studied. The ultrasonic degradation kinetics in water were found to be first-order and the degradation rate coefficient is $1.35 \times 10^{-2} \min ^{-1}(\mathrm{R}=0.9934, \mathrm{n}=8)$ at $20 \pm 1^{\circ} \mathrm{C}$. The influence of the initial concentrations, reaction temperature and the $\mathrm{pH}$ of medium on the ultrasonic decomposition of methyl violet were also investigated.
\end{abstract}

Keywords: Methyl violet, degradation kinetics, sonication, sonochemistry

\section{Introduction}

The sonochemical reaction is regarded to originate from acoustic cavitation. The cavitation processes consist of the creation, growth and implosive collapse of gas vacuoles in a solution. According to the "hot spot" theory [1], extreme temperatures $(>5000 \mathrm{~K})$ and high pressures $(>1000 \mathrm{~atm})$ occur within the bubbles during cavitational collapse. Under these extreme conditions, most organic compounds decompose in the cavitation bubbles as well as compounds present in the surrounding condensed layer undergo reactions comparable to those found in high temperature combustion. The ultrasonic degradation in aqueous solution of a large number of chemical compounds of environmental interest such as 1,1,1-trichloroethane [2], chlorinated hydrocarbons [3,4], polychlorinated biphenyls [5], phenols [6,7] and surface active reagents [8] have been studied. Dyes are an important kind of environmental pollutants in rivers or lakes. But the sonolysis of dye has not been reported before. In this paper we have studied the sonication of an aqueous solution of methyl violet that was chosen as a model of basic dye. 


\section{Results and Discussion}

An air saturated aqueous solution of methyl violet was sonicated for $120 \mathrm{~min}$. During the sonication, the concentrations of methyl violet were determined every $15 \mathrm{~min}$ and the ultraviolet absorption spectra of the aqueous solution of methyl violet were measured every 30 min during sonication. Figure 1 shows the ultraviolet absorption spectra of the solution. The degradation kinetics of methyl violet are shown in Figure 2 and Figure 3.

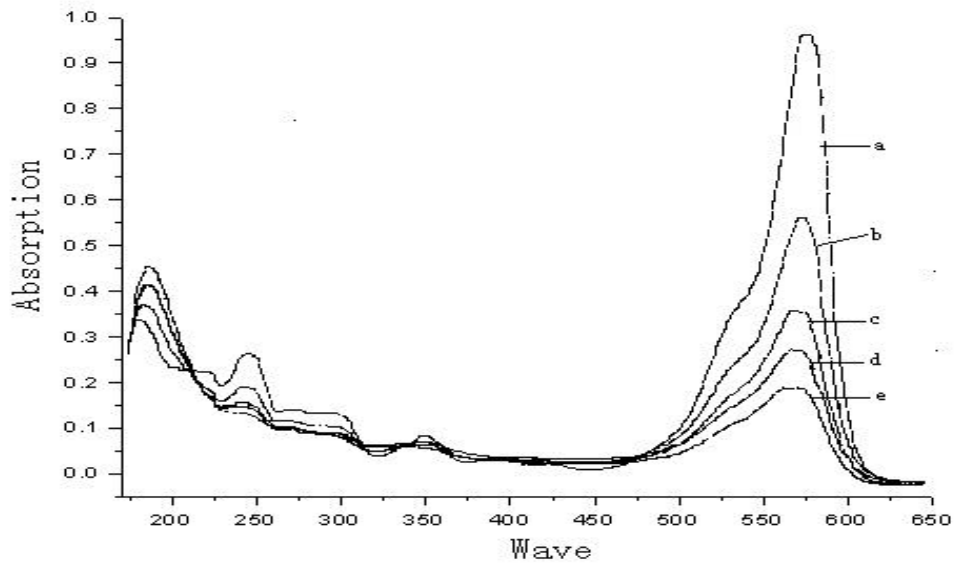

Figure 1. UV absorption spectra of aqueous solution of methyl violet at different sonication times: a. 0 min; b. $30 \mathrm{~min}$; c. $30 \mathrm{~min}$; d. $90 \mathrm{~min}$; e. $120 \mathrm{~min}$.

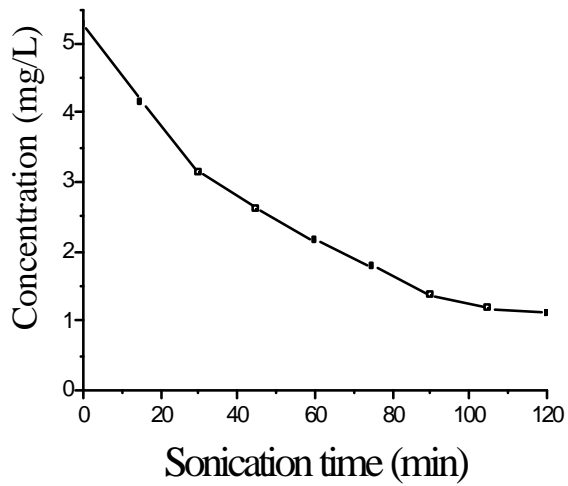

Figure 2. Concentration-sonication time profiles of methyl violet.

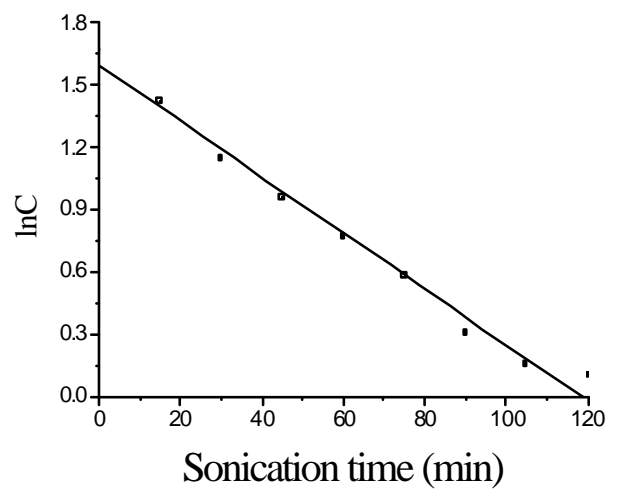

Figure 3. Degradation kinetics of methyl violet.

It was found from the results that the concentrations of methyl violet in aqueous solution decreased exponentially with sonication time, indicating first-order kinetics. Regression on the experiment data delivered the first-order reaction rate coefficient $\left(k_{1}\right)$ is $1.35 \times 10^{-2} \min ^{-1}(\mathrm{R}=0.993, \mathrm{n}=8)$ at $20 \pm 1{ }^{\circ} \mathrm{C}$.

It was also found that the ultrasonic degradation rates of methyl violet varied with different initial concentrations. Figure 4 shows the degradation kinetics of methyl violet for different initial 
concentrations at $20^{\circ} \mathrm{C}$. It was observed that as the initial concentrations increased, the degradation rate coefficients decreased. The ultrasonic degradation rate coefficients $\left(k_{1}\right)$ of methyl violet were $1.32 \times 10^{-2}$ $(\mathrm{r}=0.990, \mathrm{n}=5), 7.39 \times 10^{-3}(\mathrm{r}=0.997, \mathrm{n}=5), 5.42 \times 10^{-3}(\mathrm{r}=0.997, \mathrm{n}=5)$ and $3.57 \times 10^{-3}(\mathrm{r}=0.999, \mathrm{n}=5) \mathrm{min}^{-1}$ at initial concentrations 5.0, 10.0, 20.0 and $40.0 \mathrm{mg} \mathrm{L}^{-1}$, respectively.

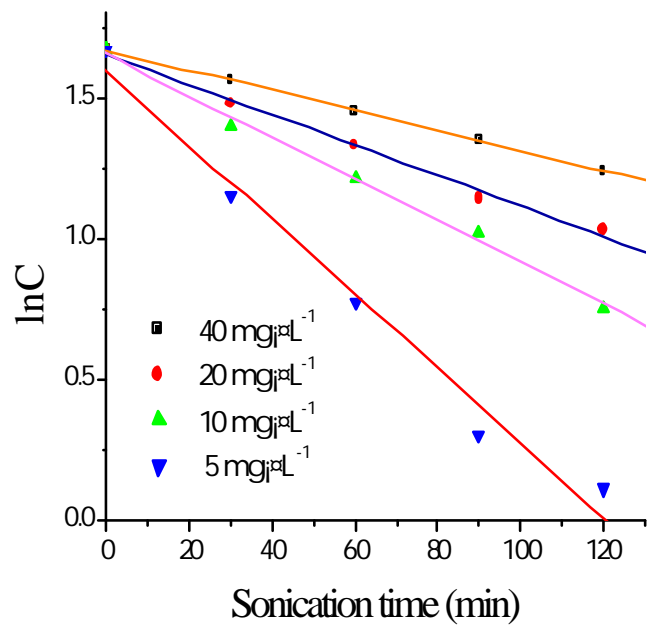

Figure 4. Degradation kinetics of methyl violet for different initial concentrations at $20^{\circ} \mathrm{C}$.

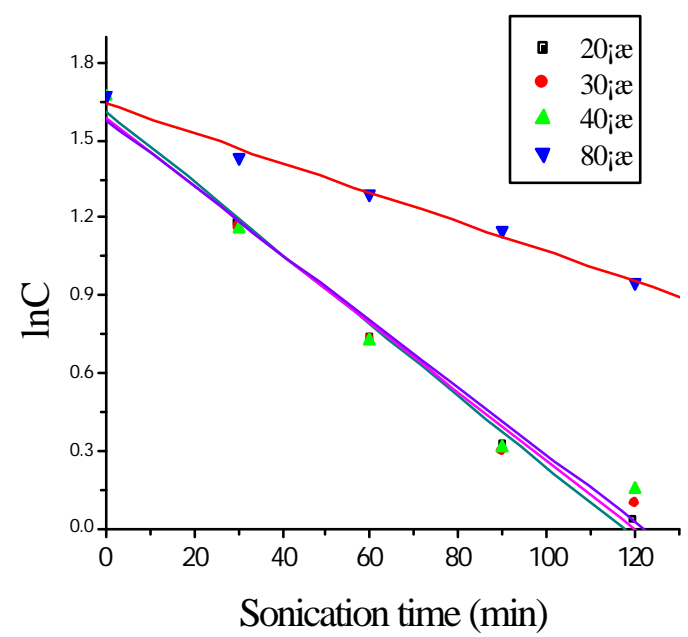

Figure 5. The influence of temperature on the ultrasonic degradation rate of methyl violet.

The influence of the temperature of the reaction solution on the ultrasonic degradation of methyl violet was investigated and the results are shown in Figure 5. It was found that the ultrasonic degradation rate coefficie nts $\left(k_{1}\right)$ of methyl violet were $1.35 \times 10^{-2}, 1.32 \times 10^{-2}, 1.28 \times 10^{-2}$ and $5.10 \times 10^{-3}$ $\min ^{-1}$ at temperature $20 \pm 1^{\circ} \mathrm{C}, 30 \pm 1^{\circ} \mathrm{C}, 40 \pm 1^{\circ} \mathrm{C}$ and $80 \pm 2^{\circ} \mathrm{C}$, respectively. The results show that the ultrasonic degradation rate was almost invariable at temperature $20-40^{\circ} \mathrm{C}$, but the ultrasonic degradation rate was decreased remarkably when the temperature of the solution rose to $80^{\circ} \mathrm{C}$. It has been reported that three different regions are formed in the aqueous sonochemical process [9]: (1) The gas phase within the cavitation bubble where elevated temperature and high pressure are produced, (2) The interfacial zone between the bubble and the bulk solution where the temperature is lower than that inside the bubble but still high enough for a sonochemical reaction. (3) The bulk solution at ambient temperature where reaction still takes place. Of the aforementioned three regions, we prefer the interfacial zone as the region where methyl violet was destructured because of the low vapor pressure of the compound. So the influence of temperature of the reaction solution on the ultrasonic degradation is not remarkable at temperature $20-40^{\circ} \mathrm{C}$. When the temperature of the solution rose to $80^{\circ} \mathrm{C}$, the number of cavitation bubbles were reduced remarkably and the ultrasonic degradation rate of methyl violet was decreased. 
The influence of the acidity of the reaction medium on the ultrasonic degradation of methyl violet was also studied and the results are shown in Figure 6. During sonication the buffers limited the $\mathrm{pH}$-variation to less than $0.4 \mathrm{pH}$-unit. So there is an influence of $\mathrm{pH}$ on the degradation rate of methyl violet. It was found that the ultrasonic degradation rate coefficients $\left(k_{1}\right)$ of methyl violet in acidic water( $\mathrm{pH} 2-4)$ are higher than those obtained in neutral aqueous solution ( $\mathrm{pH} 5-10)$ and the $k_{1}$ obtained at basic medium $(\mathrm{pH}>10)$ are the lowest.

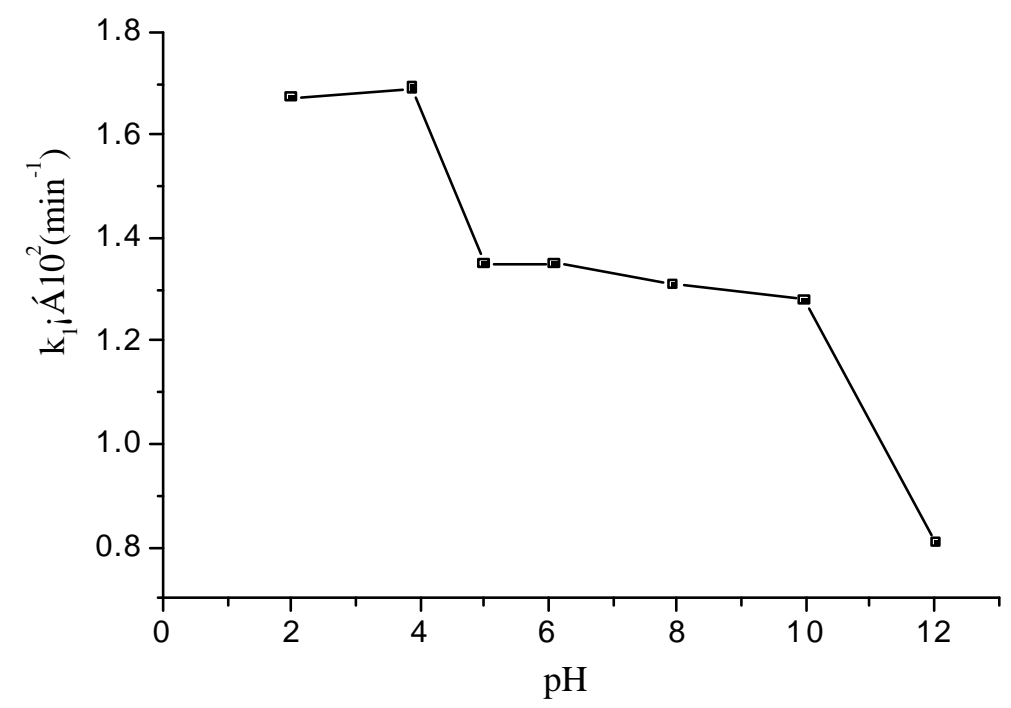

Figure 6. The influence of the acidity of reaction medium on the degradation of methyl violet.

\section{Conclusions}

The sonochemical degradation of methyl violet that was chosen as a model of basic dye in aqueous solution was studied. It was found that the ultrasonic degradation kinetics of methyl violet in water is first-order and the degradation rate coefficient is $1.347 \times 10^{-2} \mathrm{~min}^{-1}(\mathrm{R}=0.9934, \mathrm{n}=8)$ at $20 \pm 1^{\circ} \mathrm{C}$ with initial concentrations $5.0 \mathrm{mgL}^{-1}$. It was observed that with increasing initial concentration, the degradation rate coefficients decreased. The influence of the temperature of the reaction solution on the ultrasonic degradation of methyl violet was also investigated and found that the ultrasonic degradation rate was almost invariable at temperature $20-40^{\circ} \mathrm{C}$ and was decreased remarkably when the temperature of the solution rose to $80^{\circ} \mathrm{C}$. The influence of the acidity of the reaction medium on the ultrasonic degradation was that the ultrasonic degradation rate of methyl violet in acidic water are higher than those obtained in neutral aqueous solution and the $k_{1}$ obtained at basic medium $(\mathrm{pH}>10)$ are the lowest.

\section{Acknowledgments}

We thank the Natural Science Foundation of Shandong Province (No. Y98B03022) and the Foundation for University Key Teacher of the Ministry of Education (No. GG-630-11944-1016) for their financial support. 


\section{Experimental}

\section{General}

Aqueous solutions of methyl violet $\left(5 \mathrm{mgL}^{-1}\right.$ by mass) were prepared by adding methylviolet ( $5 \mathrm{mg}$ ) to deionized water $(1.0 \mathrm{~L}$ ) and stirring for $2 \mathrm{~h}$. The sonication of $100 \mathrm{~mL}$ aqueous solutions of methyl violet in a $150 \mathrm{~mL}$ glass reactor cell were performed with a $20 \mathrm{KHz}$ Model JCS-204 Ultrasonic Reactor. The aqueous solution was saturated with pure air before and during the sonication. The ultrasonic power was $50 \mathrm{~W}$. The reaction temperature was controlled with the help of condensation water surrounding the reactor cell. The quantifications of methyl violet were carried out with a model UV-3000 photometer (Shimadzu, Japan) and a model 244 HPLC (Beckman, USA).

\section{References}

1. Suslick, K.S.; Doktycs, S.J.; Flint, E.B. On the Origins of Sonochemistry and Sonoluminescence. Ultrasonics 1990, 28, 280-290.

2. Gaddam, K.; Cheung, H.M., Effects of Pressure, Temperature, and $\mathrm{pH}$ on the Sonochemical Destruction of 1,1,1-Trichloroethane in Dilute Aqueous Solution. Ultrason. Sonochem. 2001, 8, 103-109.

3. Drijvers, D.; Van Langenhove, H.; Nyugen Thi Kim, L.; Bray, L. Sonolysis of an Aqueous Mixture of Trichloroehtylene and Chlorobenzene. Ultrason. Sonochem. 1999, 6, 115-121.

4. Drijvers, D.; Van Langenhove, H.; Herrygers, V. Ultrasonic Degradation of Fluoro-, Chloro-, Bromo- and Iodobenzene: a Comparative Study. Ultrason. Sonochem. 2000, 7, 177-199.

5. Xi Kui Wang; Guan Hong Chen; Zhong Yan Yao. Sonochemical Degradation of Polychlorinated Biphenyls in Aqueous Solution. Chin. Chem. Lett., 2002, 13, in press.

6. Petrier, C.; Francony, A. Ultrasonic Waste-Water Treatment: Incidence of Ultrasonic Frequency on the Rate of Phenol and Carbon Tetrachloride Degradation. Ultrason. Sonochem. 1997, 4, 295-300.

7. Hua, I.; Höchemer, R.H.; Hoffmann, M.R. Sonochemical Degradation of p-Nitrophenol in a Parallel-Plate Near-Field Acoustical Processor. Environ. Sci. Technol., 1995, 29, 2790-1796.

8. Yan Liu, The Degradation of DBS by Sonochemistry. Chin. J. Appl. Acoust. 1999, 18(2), 35-37.

9. Riesz, P.; Kondo, T.; Murali, Krishna C Sonochemistry of Volatile and Non-volatile Solutes in Aqueous Solutions. Ultrasonics, 1990, 28, 295-303.

(C) 2003 by MDPI (http://www.mdpi.org). Reproduction is permitted for noncommercial purposes. 